\title{
Maturação fisiológica e dormência em sementes de jurema-de-embira (Mimosa ophthalmocentra)
}

\author{
Moadir de Sousa Leite ${ }^{*}$ Narjara Walessa Nogueira ${ }^{1}$ Rômulo Magno Oliveira de Freitas ${ }^{2}$ Tiago de Sousa \\ Leite $^{3}$ Pompeu Paes Guimarães ${ }^{1}$ \\ ${ }^{1}$ Universidade Federal Rural do Semi-Árido, Ave. Francisco Mota, 572 - Pres. Costa e Silva, Mossoró - RN, 59625-900 \\ 2 Instituto Federal de Educação, Ciência e Tecnologia do Ceará, Campus Crato, Rod. CE 292,15 - Crato - CE, 63115-500 \\ ${ }^{3}$ Instituto Federal de Educação, Ciência e Tecnologia de Alagoas, Campus Santana do Ipanema, Rua Domingos Acácio, 1609 - Santana do Ipanema - AL, 57500 - \\ 000
}

*Author for correspondence: moadirleite63@gmail.com

Received: January 2018 / Accepted: June 2019 / Published: June 2019

\section{Resumo}

O estudo da maturação fisiológica das sementes é uma importante forma de conhecer o comportamento das espécies vegetais quanto à reprodução, possibilitando assim, conhecer a época ideal de colheita. O estudo de aspectos físicos como tamanho e coloração das sementes, aliado a técnicas de superação de dormência, são estratégias utilizadas para obtenção de sementes com menores níveis de dormência e maior capacidade germinativa. Dessa forma, objetivou-se avaliar os diferentes estádios de maturação fisiológica com base na coloração de frutos e sementes, bem como os seus efeitos sobre a dormência, germinação e vigor de sementes de Mimosa ophthalmocentra. O trabalho foi conduzido no Laboratório de Análise de Sementes do Centro de Ciências Agrárias da Universidade Federal Rural do Semi-Árido. O delineamento estatístico utilizado foi o inteiramente casualizado, em esquema fatorial $2 \times 5$ (com e sem quebra de dormência $\mathrm{x}$ cinco estádios de maturação), constituindo assim 10 tratamentos em quatro repetições. A máxima qualidade fisiológica e vigor em sementes de Mimosa ophthalmocentra são obtidas em sementes da classe de coloração marrom clara, desde que essas passem por tratamentos pré-germinativos.

Palavras-chave: Época de colheita, Vigor, Caatinga.

\section{Abstract}

Studying seed physiological maturation is an important way to know the behavior of plant species in terms of reproduction, thus enabling us to know the ideal harvest season. The study of physical aspects such as seed size and color, allied to techniques to overcome dormancy, are strategies used to obtain seeds with lower levels of dormancy and higher germination capacity. Therefore, this study aimed to evaluate the different stages of physiological maturation based on the color of fruits and seeds, as well as their effects on the dormancy, germination and vigor of Mimosa ophthalmocentra seeds. The work was carried out in the Laboratory of Seed Analysis at the Center of Agricultural Sciences of the Federal Rural University of the Semi-Arid Region. A completely randomized design was used, in a $2 \times 5$ factorial scheme (with and without dormancy breaking $\times$ five maturation stages), thus totaling 10 treatments in four replications. The maximum physiological quality and vigor in Mimosa ophthalmocentra seeds are obtained in seeds of the light brown color class, provided they undergo pre-germination treatments.

Keywords: Harvest season, Vigor, Caatinga. Introdução
A Caatinga caracteriza-se pela predominância de vegetação xerófila essencialmente extrativista, o que pode, em alguns casos, comprometer a biodiversidade e causar problemas ambientais. Dessa forma, estudos básicos sobre as espécies que compõem este bioma são essenciais para ampliar o conhecimento de tais espécies para manutenção da biodiversidade e composição do bioma (Lopes et al. 2014).

A jurema-de-embira (Mimosa ophthalmocentra Mart. ex Benth.) é uma espécie comum na Caatinga (Cavalcanti et al. 2009), presente em matas ciliares e de regeneração (Sampaio et al. 2010), sendo considerada uma espécie de elevado potencial madeireiro para o Nordeste (Nogueira et al. 2018). Brito et al. (2014) constataram que as sementes de Mimosa ophthalmocentra apresentam dormência tegumentar, fator que dificulta a produção de mudas e utilização dessas espécies em programas de reflorestamento e exploração econômica, visto que sua propagação é via seminífera.

Segundo Carvalho e Nakagawa (2012), a impermeabilidade do tegumento está associada a diversas espécies botânicas, sendo mais frequente naquelas da família Fabaceae, a exemplo de Mimosa ophthalmocentra. Dessa forma, o tegumento impermeável a passagem de água impede que a semente absorva água e reative os processos metabólicos da germinação, mesmo que em condições ambientais favoráveis.

Estudos mostram que sementes que desenvolvem tegumentos impermeáveis à água são capazes de embeber e germinar quando coletadas no ponto de maturidade fisiológica, antes do início da fase de dessecamento ao final da maturação (Cardoso 2009). Entretanto, estes estudos ainda são bastante escassos, sobretudo em relação à propagação de espécies da Caatinga que apresentam dormência como fator de impedimento do processo germinativo.

O processo de maturação das sementes é caracterizado por uma série de alterações morfológicas, fisiológicas e funcionais, como aumento de tamanho, variações no teor de água, vigor e acúmulo de massa seca, que ocorrem a partir da fecundação do óvulo e continuam até se atingir a maturidade fisiológica (Hehenberger et al. 2012), momento em que as sementes adquirem o máximo de matéria seca, germinação e vigor (Marcos Filho 2015).

Segundo Mata et al. (2013), ao estudar-se o processo de maturação de frutos e sementes deve-se levar em consideração as modificações de natureza morfológica e fisiológica, a exemplo do acúmulo de matéria seca, teor de água e potencial germinativo. Já Farias et al. (2011), ressaltam que em campo, a seleção dos frutos é baseada 
principalmente em características visuais, como cor do fruto, tamanho e abertura espontânea. Assim, é importante que sejam realizadas associações entre esses índices de maturação, a fim de se avaliar possíveis correlações entre as características morfológicas e a maturidade físiológica, possibilitando a definição de estratégias de colheita de sementes.

A coloração do fruto associada à redução do teor de água das sementes se mostrou um índice eficaz para determinação da maturidade fisiológica de sementes de várias espécies, como Caesalpinia echinata Lam. (Aguiar et al. 2007), Erythrina variegata L. (Matheus et al. 2011), Mimosa caesalpiniifolia Benth. (Nogueira et al. 2013), Amburana cearenses A. C. Smith (Lopes et al. 2014) e Calophyllum brasiliense Cambess (Silva et al. 2018). Assim sendo, pesquisas relacionadas ao processo de maturação das sementes são de vital importância para se determinar o ponto ideal da colheita destas, garantindo assim máxima produção e elevada qualidade fisiológica (Nogueira et al. 2013).

Dessa forma, objetivou-se avaliar os diferentes estádios de maturação fisiológica com base na coloração de frutos e sementes e os seus efeitos sobre a dormência, germinação e vigor de sementes de Mimosa ophthalmocentra.

\section{Material e Métodos}

O experimento foi conduzido no Laboratório de Análises de Sementes (LAS) do Centro de Ciências Agrárias da Universidade Federal Rural do Semi-Árido (UFERSA), situada em Mossoró-RN. Utilizaram-se frutos e sementes de Mimosa ophthalmocentra, coletadas manualmente no Museu vivo do Semi-Árido (MUVISA) UFERSA, região circunscrita às coordenadas geográficas $5^{\circ} 11^{\prime}$ de latitude sul, $37^{\circ} 20^{\prime}$ de longitude W. Gr.

As vagens foram coletadas em 20 plantas matrizes e classificadas em cinco diferentes classes de coloração: 1 vagem verde; 2 - vagens verde/amarela; 3 - vagem marrom/amarela; 4 - vagem marrom clara e 5 - vagem marrom escura (Figura 1).

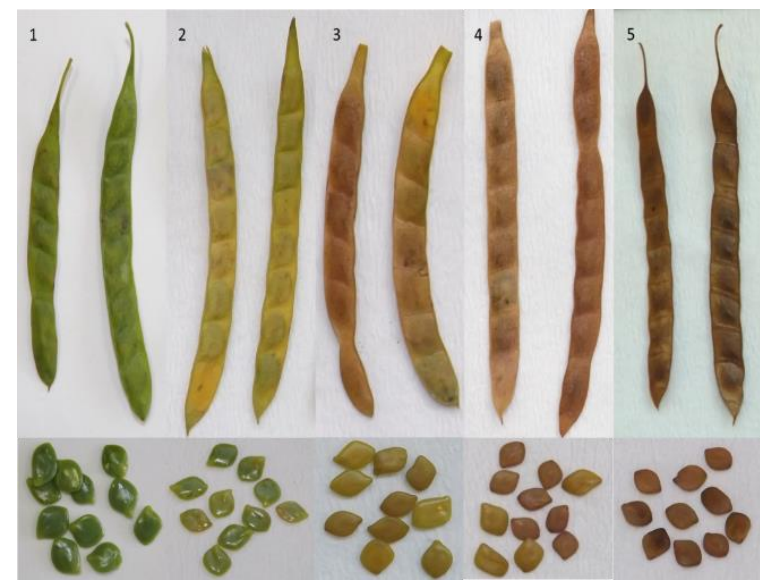

Figura 1. Diferentes estádios de maturação de frutos e sementes de jurema-de-embira (Mimosa ophthalmocentra Mart. ex Benth.) em função de diferentes classes de coloração de frutos.

As vagens foram inicialmente levadas ao Laboratório de Análise de Sementes da Universidade Federal Rural do Semi-Árido, onde foram beneficiadas de forma manual, eliminando-se aquelas atacadas por pragas e doenças. Em seguida, procedeu-se com a debulha manual das mesmas para a retirada das sementes.
Para avaliação do potencial germinativo, foram adotados tratamentos com e sem superação de dormência, de forma que houvesse a possibilidade da determinação do início da dormência nas sementes. O tratamento prégerminativo foi aplicado por meio de desponte, sendo este realizado através do corte do tegumento das sementes no lado oposto ao ponto de inserção na vagem.

Para a execução do teste de germinação, utilizou-se quatro repetições de 25 sementes, dispostas em rolo de papel, contendo três folhas de papel tipo germitest $\AA$ como substrato. Anteriormente a semeadura, o substrato foi umedecido com água destilada com quantidade equivalente a 2,5 vezes o peso do papel sem hidratação, visando uniformização do teste. Após a semeadura os rolos foram acondicionados em sacos plásticos e transferidos para câmara de germinação tipo B.O.D. (Biochemical Oxygen Demand) com temperatura constante de $30^{\circ} \mathrm{C}$ e fotoperíodo de $8 \mathrm{~h} / \mathrm{dia}$.

As características avaliadas foram:

Teste de germinação e primeira contagem de germinação: realizados conjuntamente, sendo a primeira contagem de germinação realizada aos três dias e a germinação final contabilizada aos sete dias após a instalação do teste, por ocasião da estabilização do estande, sendo determinado o número de plântulas normais. As taxas germinativas aos três e sete dias foram obtidas pela relação entre o número de plântulas normais germinadas/número total de sementes x 100 .

Índice de velocidade de germinação: realizado concomitantemente ao teste de germinação, com contagem diária do número de plântulas germinadas até o sétimo dia após a instalação do teste de germinação, procedendo os cálculos de acordo com a fórmula proposta por Maguire (1962).

Teor de água de frutos e sementes: para o teor de água de frutos, tomou-se duas amostras contendo 20 frutos cada, enquanto que o teor de água das sementes foi determinado em duas amostras contendo 20 sementes cada. As amostras foram pesadas inicialmente em balança analítica de precisão (peso úmido), sendo posteriormente submetidas à secagem pelo método da estufa, a $105^{\circ} \mathrm{C} \pm 3$ por 24 horas, de acordo com as Regras para Análise de Sementes (Brasil 2009). Após o período de secagem, as mesmas foram novamente pesadas (peso seco), sendo então determinado o teor de água, com os resultados expressos em porcentagem, não sendo comparados estatisticamente.

Peso fresco e seco de frutos e sementes: para o peso fresco de frutos, selecionou-se duas amostras contendo 20 frutos cada, enquanto que para o peso fresco de sementes, utilizou-se duas amostras contendo 20 sementes cada, sendo estas pesadas inicialmente em balança analítica de precisão, com os resultados expressos em mg. Para a determinação do peso seco, os mesmos frutos e sementes utilizadas para determinação do peso fresco foram postos em estufa a $105 \pm 3^{\circ} \mathrm{C}$ por 24 horas (Brasil 2009). Posteriormente, o peso seco de frutos e sementes foi aferido em balança analítica de precisão, com os resultados expressos em mg.

Utilizou-se o delineamento estatístico inteiramente casualizado (DIC), em esquema fatorial $2 \times 5$ (com e sem quebra de dormência x cinco estádios de maturação), contendo 10 tratamentos, com quatro repetições cada.

Preliminarmente, testou-se a normalidade e a igualdade de variâncias, pelos testes de KolmogorovSmirnov $(\mathrm{P}<0,01)$ e Bartlett $(\mathrm{P}<0,01)$, respectivamente. Em seguida, os resultados foram submetidos à análise de variância pelo teste $\mathrm{F}$ a $5 \%$ de probabilidade, com o auxílio 
Leite et al

do programa Assistat (Silva e Azevedo 2016). A comparação entre as médias foi feita utilizando-se o teste de Tukey a $5 \%$ de probabilidade.

\section{Resultados}

Verificou-se a influência da dormência na germinação em todas as classes de coloração, onde ocorreram diferenças significativas entre as sementes sem tratamento e aquelas submetidas a tratamento pré-germinativo, sendo os maiores percentuais de germinação obtidos com as sementes submetidas ao desponte (Tabela 1).

Tabela 1. Germinação de sementes de jurema-de-embira (Mimosa ophthalmocentra Mart. ex Benth.) com e sem tratamento prégerminativo em função de diferentes classes de coloração de frutos.

\begin{tabular}{|c|c|c|c|c|c|}
\hline \multirow{3}{*}{ Tratamento } & \multicolumn{5}{|c|}{ Classes de coloração } \\
\hline & Verde & Verde/Amarela & Amarela/Marrom & Marrom clara & Marrom escura \\
\hline & (1) & (2) & (3) & (4) & (5) \\
\hline Sem desponte & $50 \mathrm{bB}$ & $73 \mathrm{bA}$ & $77 \mathrm{aA}$ & $54 \mathrm{bB}$ & $6 \mathrm{bC}$ \\
\hline Com desponte & $56 \mathrm{aC}$ & $80 \mathrm{aA}$ & $82 \mathrm{aA}$ & $86 \mathrm{aA}$ & $69 \mathrm{aB}$ \\
\hline Fonte de & \multirow{2}{*}{\multicolumn{5}{|c|}{ Valores de $\mathrm{F}$}} \\
\hline Variação & & & & & \\
\hline $\mathrm{T} \times \mathrm{C}$ & \multicolumn{5}{|c|}{$81,71^{* * *}$} \\
\hline C.V. (\%) & \multicolumn{5}{|c|}{6,24} \\
\hline
\end{tabular}

*As médias seguidas pela mesma letra maiúscula na linha e minúscula na coluna não diferem estatisticamente entre si pelo teste de Tukey ao nível de 5\% de probabilidade.

Os melhores resultados para a germinação foram obtidos nas classes de coloração verde/amarela (2), amarela/marrom (3) e marrom clara (4), e com sementes que passaram por processos de quebra de dormência, sendo a classe 4 a que apresentou maior germinação, embora estatisticamente igual à verificada para as classes 2 e 3. Para as sementes sem quebra de dormência, a maior taxa de germinação foi obtida na classe de coloração amarela/marrom (3), não diferindo estatisticamente dos resultados encontrados para a classe verde/amarela (2).

Para o tratamento sem desponte, os maiores valores de primeira contagem de germinação coincidiram com os resultados verificados no teste de germinação, sendo obtidos para a classe amarela/marrom (3), onde este valor foi estatisticamente superior aos encontrados para as classes verde (1), verde/amarela (2), marrom clara (4) e marrom escura (5) (Tabela 2). Para o tratamento com desponte, o maior valor para germinação na ocasião da primeira contagem foi obtido para a classe de coloração 4 , mas que não diferiu estatisticamente das classes 2 e 3 .

Tabela 2. Primeira contagem de germinação de sementes de jurema-de-embira (Mimosa ophthalmocentra Mart. ex Benth.) com e sem tratamento pré-germinativo em função de diferentes classes de coloração de frutos.

\begin{tabular}{|c|c|c|c|c|c|}
\hline \multirow{3}{*}{ Tratamento } & \multicolumn{5}{|c|}{ Classes de coloração } \\
\hline & Verde & Verde/Amarela & Amarela/Marrom & Marrom clara & Marrom escura \\
\hline & (1) & (2) & (3) & (4) & (5) \\
\hline Sem desponte & $45 \mathrm{bC}$ & $62 \mathrm{bB}$ & $77 \mathrm{aA}$ & $30 \mathrm{bD}$ & $6 \mathrm{bE}$ \\
\hline Com desponte & $56 \mathrm{aC}$ & $80 \mathrm{aA}$ & $82 \mathrm{aA}$ & $86 \mathrm{aA}$ & $69 \mathrm{aB}$ \\
\hline Fonte de & & & & & \\
\hline Variação & & & Valor de F & & \\
\hline $\mathrm{T} \times \mathrm{C}$ & & & $86,80^{* * *}$ & & \\
\hline C.V. (\%) & & & 6,88 & & \\
\hline
\end{tabular}

*Médias seguidas pela mesma letra maiúscula na linha e minúscula na coluna não diferem estatisticamente entre si pelo teste de Tukey ao nível de 5\% de probabilidade.

Para a classe amarela/marrom (3), não houve diferenças estatísticas entre os tratamentos com e sem desponte, sendo este o único estádio de maturação em que as sementes com tratamento pré-germinativo não apresentaram resultado superior as sementes sem tratamento pré-germinativo.

Para as sementes que não passaram por tratamento prégerminativo, o índice de velocidade de germinação teve comportamento similar à germinação e a primeira contagem de germinação, sendo que os maiores valores para essa variável, em sementes sem desponte, foram obtidos em sementes da classe amarela/marrom (3) (Tabela 3).

Tabela 3. Índice de velocidade de germinação de sementes de jurema-de-embira (Mimosa ophthalmocentra Mart. ex Benth.) com e sem tratamento pré-germinativo em função de diferentes classes de coloração de frutos.

\begin{tabular}{cccccc}
\hline \multirow{2}{*}{ Tratamento } & \multicolumn{5}{c}{ Classes de coloração } \\
\cline { 2 - 6 } & $\begin{array}{c}\text { Verde } \\
\text { (1) }\end{array}$ & $\begin{array}{c}\text { Verde/Amarela } \\
\text { (2) }\end{array}$ & $\begin{array}{c}\text { Amarela/Marrom } \\
\text { (3) }\end{array}$ & $\begin{array}{c}\text { Marrom clara } \\
\text { (4) }\end{array}$ & $\begin{array}{c}\text { Marrom escura } \\
(5)\end{array}$ \\
\hline Sem desponte & $2,30 \mathrm{aB}$ & $6,01 \mathrm{bA}$ & $8,46 \mathrm{aA}$ & $1,65 \mathrm{bB}$ & $0,35 \mathrm{bB}$ \\
Com desponte & $2,74 \mathrm{aD}$ & $8,91 \mathrm{aC}$ & $9,82 \mathrm{aC}$ & $12,49 \mathrm{aB}$ & $15,16 \mathrm{aA}$ \\
\hline Fonte de & \multicolumn{5}{c}{ Valor de F } \\
Variação & \multicolumn{5}{c}{$57,38^{* *}$} \\
\hline T x C & 17,55 \\
\hline C.V. (\%) & \multicolumn{5}{c}{} \\
\hline
\end{tabular}

*As médias seguidas pela mesma letra maiúscula na linha e minúscula na coluna não diferem estatisticamente entre si pelo teste de Tukey ao nível de 5\% de probabilidade.

Para as sementes que passaram por tratamento prégerminativo, o maior valor para o índice de velocidade de germinação foi obtido em sementes da classe de coloração marrom escura (5). Nessa classe, em que as sementes com desponte apresentaram maior velocidade de germinação, as sementes sem quebra de dormência apresentaram os piores resultados para esse índice.

O teor de água nos frutos e nas sementes apresentou decréscimo contínuo entre as diferentes classes de coloração (Tabela 4). O máximo teor de água nos frutos e nas sementes foi de 62,2 e $68,26 \%$, respectivamente, obtido em vagens da classe verde (1), enquanto que o menor teor de água nos frutos e nas sementes foi de 7,22 e $6,17 \%$, respectivamente, obtido em vagens da classe marrom escura (5).

Os dados referentes ao peso fresco de frutos e sementes apresentaram redução linear (Tabela 1). O maior valor para o peso fresco de frutos $(195,2 \mathrm{mg})$ e sementes $(9,7 \mathrm{mg})$ foi obtido na classe de coloração verde (1), enquanto que os menores valores para frutos $(79,2 \mathrm{mg}) \mathrm{e}$ sementes $(3,2 \mathrm{mg})$ foram obtidos na classe marrom escura (5).

Tabela 4. Teor de água de frutos (TAF) e sementes (TAS), peso fresco de frutos (PFF) e sementes (PFS) e peso seco de frutos (PSF) e sementes (PSS) de jurema-de-embira (Mimosa ophthalmocentra Mart. ex Benth.) em função de diferentes classes de coloração de frutos.

\begin{tabular}{|c|c|c|c|c|c|c|}
\hline \multirow{2}{*}{ Classes } & TAF & TAS & PFF & PFS & PSF & PSS \\
\hline & \multicolumn{2}{|r|}{-} & & & $\mathrm{mg}$ & 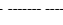 \\
\hline Verde & 62,2 & 68,26 & $195,2 \mathrm{a}$ & $9,7 \mathrm{a}$ & $73,8 \mathrm{~b}$ & $3,1 \mathrm{c}$ \\
\hline Verde/Amarela & 56,44 & 62,9 & $178,6 \mathrm{a}$ & $9,6 \mathrm{a}$ & 77,8 ab & $3,6 \mathrm{~b}$ \\
\hline Amarela/Marrom & 41,63 & 40,85 & $140,7 \mathrm{~b}$ & $7,3 \mathrm{~b}$ & 82,1 ab & $4,3 \mathrm{a}$ \\
\hline Marrom clara & 7,82 & 17,69 & $97,3 \mathrm{c}$ & $4,6 \mathrm{c}$ & $89,7 \mathrm{a}$ & $3,8 \mathrm{~b}$ \\
\hline Marrom escura & 7,22 & 6,17 & $79,2 \mathrm{~d}$ & $3,2 \mathrm{~d}$ & $73,5 \mathrm{~b}$ & $3,0 \mathrm{c}$ \\
\hline $\mathrm{F}$ & - & & $77,02^{* * *}$ & $166,3^{* * *}$ & $4,78^{*}$ & $24,61^{* *}$ \\
\hline C.V. $(\%)$ & - & - & 8,26 & 6,56 & 7,76 & 6,29 \\
\hline
\end{tabular}

*Médias seguidas pela mesma letra minúscula na coluna não diferem estatisticamente entre si pelo teste de Tukey ao nível de $5 \%$ de probabilidade.O peso seco de frutos e sementes apresentou comportamento quadrático (Tabela 
1), sendo o maior peso de frutos $(89,7 \mathrm{mg})$ encontrado na classe de coloração marrom clara (4). Já em relação as sementes, o maior peso seco $(4,3 \mathrm{mg})$ foi obtido em sementes pertencentes a classe de coloração amarela/marrom (3)

\section{Discussão}

Os resultados obtidos para germinação corroboram os encontrados por Muller et al. (2016), que verificaram os menores percentuais de germinação em sementes de Peltophorum dubium (Spreng) Taub. quando estas estavam completamente maduras, época em que o tegumento da maioria delas se encontrava completamente impermeável, impedindo, assim, a entrada de água.

Segundo Bewley et al. (2013), a causa mais comum de dormência em sementes de plantas da família Fabaceae é a impermeabilidade do tegumento, caracterizada como dormência exógena-física, onde os envoltórios conferem determinada resistência à entrada de água e/ou gases ao embrião, em decorrência de serem constituídos por um conjunto de células esclerenquimáticas contendo substâncias hidrofóbicas, dificultando o início da sua hidratação e consequentemente interferindo nas reações metabólicas básicas da germinação.

Trabalhando com maturação fisiológica e dormência de sementes de Mimosa caesalpiniifolia Benth., Nogueira et al. (2013) verificaram que para sementes sem tratamento pré-germinativo na ocasião da primeira contagem, houve um leve aumento na germinação ao longo dos estádios de maturação estudados, sendo seguido por uma queda acentuada. Para o tratamento com quebra de dormência, resultados semelhantes foram verificados, havendo uma queda na germinação da classe marrom escura (5), onde o tegumento já estava completamente impermeável.

A perda acentuada de água e a instalação da dormência nos estádios finais da maturação são consideradas como uma estratégia de preservação das espécies, permitindo a dispersão espacial das sementes e a distribuição temporal da germinação, em condições muitas vezes não favoráveis (Marcos Filho, 2015).

De acordo com Marcos Filho (2015), durante o início do desenvolvimento, as sementes possuem um requerimento maior de água em função da intensa divisão, alongamento celular e alocação de compostos orgânicos, sendo sucedido pelo processo de dessecação, que segundo Carvalho e Nakagawa (2012), é comum em espécies florestais, como Anadenanthera colubrina (Vellozo) Brenan (Pires Neto et al. 2016), Allophylus edulis [(A. St.Hil., A. Juss. \& Cambess.) Hieron. ex Niederl.] (Kaiser et al. 2016), Moringa oleifera Lam. (Agustine et al. 2015) e Peltophorum dubium (Spreng) Taub. (Muller et al. 2016), onde o teor de água decresceu continuadamente ao longo da maturação das sementes.

O acúmulo de massa seca é descrito por Carvalho e Nakagawa (2012), como sendo um processo inicialmente lento, pois está predominando processos de divisão e expansão celulares. Após esta fase, o acúmulo de matéria seca é intensificado até atingir o máximo, quando as sementes se desligam fisiologicamente da planta-mãe. Os mesmos autores relatam ainda que o vigor de uma semente, durante a maturação, é uma característica que acompanha, de maneira geral e na mesma proporção, o acúmulo de massa seca. Dessa forma, o máximo vigor de uma semente é atingido por ocasião do máximo acúmulo de massa seca, sugerindo então, que a maturidade fisiológica de sementes de jurema-de-embira ocorre em vagens amarelas/marrons (estádio 3), podendo haver defasagens entre as curvas, em função das condições ambientais.

O atraso da colheita a partir do ponto de maturidade fisiológica acarreta sérios problemas na quantidade e na qualidade das sementes produzidas (Nakagawa et al. 2010). Esses problemas são determinados pela exposição das sementes a condições desfavoráveis do ambiente, uma vez que a colheita não é indicada no ponto de maturidade fisiológica, devido o elevado grau de umidade da semente, o que gera dificuldades para recolhimento e debulha (quantidade excessiva de partes verdes e úmidas das plantas) e ocorrência de injúrias mecânicas por amassamento das sementes, fato este que causa imensas perdas na quantidade e qualidade das sementes.

Dessa forma, sugere-se que a instalação da dormência em sementes de Mimosa ophthalmocentra ocorre na classe de coloração marrom clara (4), devido a redução dos índices avaliados por ocasião da passagem da classe amarela/marrom (3) para a classe marrom clara (4), o que dificulta o manuseio das sementes da espécie, trazendo a necessidade de aplicação de métodos pré-germinativos, como o desponte.

\section{Conclusões}

As sementes de Mimosa ophthalmocentra apresentam dormência tegumentar, tornando-se mais intensa em sementes provenientes de vagens marrom-claras (classe 4), sendo necessária a aplicação de técnicas de superação de dormência para acelerar e uniformizar a germinação.

A máxima qualidade fisiológica e vigor em sementes de Mimosa ophthalmocentra são obtidas em sementes da classe de coloração marrom clara, desde que essas passem por tratamentos pré-germinativos.

\section{Referências}

Aguiar FFA, Pinto MM, Tavares AR, Kanashiro S (2007) Maturação de frutos de Caesalpinia echinata Lam., paubrasil. Revista Árvore, 31 (1): 1-6. http://dx.doi.org/10.1590/S0100-67622007000100001

Agustini MB, Wendt L, Paulus C, Malavasi MM, Gusatto FC (2015) Maturidade fisiológica de sementes de Moringa oleifera (Lam). Revista Cultivando o Saber, 8 (3): 267278.

Bewley JD, Bradford K, Hilhorst H, Nonogaki H (2013) Seeds: physiology of development, germination and dormancy. $3^{\text {th }}$ Edition. New York: Springer. 392p.

BRASIL. Ministério da Agricultura, Pecuária e Abastecimento (2009) Regras para análise de sementes. Brasília: Mapa/ACS. 395p.

Brito AS, Pinto MADSC, Araújo AV, Souza VN (2014) Superação de dormência em Mimosa ophthalmocentra Mart. ex Benth. Enciclopédia biosfera, 10 (18): 27922800 .

Cardoso VJM (2009) Conceito e classificação da dormência em sementes. Oecologia Brasiliensis, 13 (4): 619-631. doi:10.4257/oeco.2009.1304.06

Carvalho NM, Nakagawa J (2012) Sementes: ciência, tecnologia e produção. $5^{\text {th }}$ Edition. Jaboticabal: Funep. 590 p.

Cavalcanti ADC, Rodal MJN, Sampaio EVSB, Costa KCC (2009) Mudanças florísticas e estruturais, após cinco anos, em uma comunidade de Caatinga no Estado de Pernambuco, Brasil. Acta Botanica Brasilica, 23 (4): 


$\begin{array}{ll}\text { 1210-1212. } & \text { http://dx.doi.org/10.1590/S0102- } \\ 33062009000400032 & \end{array}$

33062009000400032

Farias JF, Araújo Neto SE, Álvares VS, Ferraz PA, Furtado DT, Souza ML (2011) Maturação e determinação do ponto de colheita de frutos de envira-caju. Revista Brasileira de Fruticultura, 33 (3): 730-736.

Hehenberger E, Kradolfer D, Köhler C (2012) Endosperm cellularization defines an important developmental transition for embryo development. Development, 139 (11): 2031-2039. doi: 10.1242/dev.077057

Kaiser DK, Freitas LCN, Dranski JAL, Malavasi MM, Malavasi UC, Kosmann CR, Andrioli KK (2016) Physiological maturity of seeds and colorimetry of the fruits of Allophylus edulis [(A. St.-Hil., A. Juss. \& Cambess.) Hieron. ex Niederl.]. Journal of Seed Science, 38 (2): 92-100. http://dx.doi.org/10.1590/2317$1545 \mathrm{v} 38 \mathrm{n} 1154590$

Lopes IS, Nóbrega AMF, Matos VP (2014) Maturação e colheita da semente de Amburana cearensis (Allem.) A. C. Smith. Ciência Florestal, 24 (1): 565-572. http://dx.doi.org/10.5902/1980509815734

Maguire JD (1962) Speed of germination-aid in selection and evaluation for seedling emergence and vigor. Crop Science, 2 (1): 176-177.

Marcos-Filho J (2015) Fisiologia de sementes de plantas cultivadas. $2^{\text {th }}$ Edition. Londrina: Abrates. 660p.

Mata MF, Silva KB, Bruno RLA, Felix LP, Medeiros Filho S, Alves EU (2013) Maturação Fisiológica de Sementes de ingazeiro (Inga striata Benth.). Semina: Ciências Agrárias, 34 (2): 549-566. doi: 10.5433/1679$\underline{0359.2013 v 34 n 2 p 549}$

Matheus MT, Lopes JC, Corrêa NB (2011) Maturação fisiológica de sementes de Erythrina variegata L. Ciência Florestal, 21 (4): 619-627. doi: 10.5902/198050984507

Müller EM, Gibbert P, Binotto T, Kaiser DK, Bortolini MF (2016) Maturação e dormência em sementes de Peltophorum dubium (Spreng) Taub. de diferentes árvores matrizes. Iheringia Série Botânica, 71 (3): 222-229.

Nakagawa J, Mori ES, Pinto CS, Fernandes KHP, Seki MS, Meneghetti RA (2010) Maturação e secagem de sementes de Peltophorum dubium (Spreng.) Taubert (CANAFÍSTULA). Revista Árvore, 34 (1): 49-56. http://dx.doi.org/10.1590/S0100-67622010000100006

Nogueira NW, Ribeiro MCC, Freitas RMO, Martins HVG, Leal CCP (2013) Maturação fisiológica e dormência em sementes de sabiá (Mimosa caesalpiniifolia Benth.). Bioscience Journal, 29 (4): 876-883.

Nogueira NW, Torres SB, Freitas RMO, Santos PCS, Sá FVS, Leite MS (2018) Salt stress and temperatures on the germination and initial growth of 'jurema-de-embira' (Mimosa ophthalmocentra) seedlings. Revista Brasileira de Engenharia Agrícola e Ambiental, 22 (4): 273-278. http://dx.doi.org/10.1590/1807-

1929/agriambi.v22n4p273-278

Pires Neto PAF, Pires VCM, Moraes CB, Oliveira LM, Portella ACF, Nakagawa J (2016) Physiological ripening of Anadenanthera colubrina (Vellozo) Brenan seeds. Journal of Seed Science, 38 (2): 155-161. http://dx.doi.org/10.1590/2317-1545v38n2153112
Sampaio E, Gasson P, Baracat A, Cutler D, Pareyn F, Lima KC (2010) Tree biomass estimation in regenerating areas of tropical dry vegetation in northeast Brazil. Forest Ecology and Management, 259 (11): 1135-1140. https://doi.org/10.1016/j.foreco.2009.12.028

Silva FAS, Azevedo CAV (2016) The Assistat Software Version 7.7 and its use in the analysis of experimental data. African Journal Agricultural Research, 11 (39): 37333740. doi: 10.5897/AJAR2016.11522

Silva RC, Vieira ESN, Panobianco M (2018) Morphophysiological characteristics of guanandi fruit and seeds during ripening and harvesting time. Pesquisa Agropecuária Brasileira, 53 (2): 212-220. doi: $\underline{10.1590 / \mathrm{S} 0100-204 \mathrm{X} 2018000200010}$ 